\title{
SOME TOPOLOGICAL PROPERTIES OF THE FUNCTION $n(y)^{1}$
}

\section{S. SAWYER}

1. Introduction. For a continuous function $f(x)$ on the unit interval, let $n(y)$ be the function ${ }^{2}$ which counts the number of solutions of the equation $f(x)=y$-i.e., the number of intersections of the graph of $f(x)$ with the line $y_{1}=y$. The function $n(y)$ was introduced by Banach [1], who proved that $f(x)$ is of bounded variation if and only if $n(y)$ is Lebesgue integrable, and derived the formula

$$
V(f)=\int n(y) d y
$$

for the total variation of $f(x)$. In particular, if $f(x)$ is of bounded variation it has an a.e. finite $n(y)$, but in general we could have $n(y)=\infty$ identically in the range of $f(x)$ (see e.g. §3). The condition $T_{1}$ that $n(y)<\infty$ a.e. has many real variable characterizations ([2], [4, pp. 278-287]); for example, it is equivalent to certain differentiability conditions on $f(x)$. Nina Bary [2] has also shown that any continuous function on the unit interval can be written as the sum of three continuous functions satisfying $T_{1}$. The paper [3] is in a slightly different vein, in which an a.e. equivalent definition of $n(y)$ is given with applications in Fourier series. For example (assuming $f(0)=f(1)$ ), the condition

$$
\int \log ^{+} n(y) d y<\infty
$$

is shown to imply the uniform convergence of the Fourier series of $f(x)$.

The results quoted above are all applications of measure-theoretic arguments to the function $n(y)$; the purpose of this paper is to look at implications of the topological properties of $n(y)$. For example, assume $f(x)$ is a continuous function on the unit interval with the property that $n(y)<\infty$ for every $y$. Then, it follows from Theorem 1 that $f(x)$ is of bounded variation on a subinterval of any given inter-

Received by the editors October 15, 1965.

1 The above results were obtained while the author was at the California Institute of Technology and the Courant Institute of Mathematical Sciences, the latter while under Grant NSF-GP-3465.

${ }^{2}$ See $\$ 2$ for a more precise definition. 
val $(a, b) \subseteq[0,1]$. This is a much stronger result than would be possible if just $n(y)<\infty$ a.e. Also, this property is preserved under finite sums: hence, the result of Nina Bary is no longer true if $T_{1}$ is strengthened to $n(y)<\infty$, every $y$. We also remark that if $n(y)$ is everywhere finite, the Fourier series of $f(x)$ then converges uniformly at each point of an open dense set in the unit interval; this, too, is false if we merely assume $n(y)<\infty$ a.e.

$\S 3$ is devoted to some results of a negative nature. For example, it is shown by the method of category that there exist continuous functions $f(x)$ such that $n(y)=\infty$ identically for $\min f(x)<y<\max f(x)$. In fact, the function $f(x)$ can even be chosen to satisfy a Hölder condition for an arbitrary exponent $\beta<1$. Thus, for a general $y_{0}$ in the range, $f(x)$ oscillates through the line $y=y_{0}$ infinitely of ten; we also show that the amplitudes of these oscillations cannot die out too quickly. More precisely, let $y<\alpha$ be a pair of real numbers, and $0<x_{1}<x_{2}<\cdots<x_{N}<1$ a partition of $[0,1]$. Assume further that $f\left(x_{k}\right)=y$ for each $k$, and that $f(x)=\alpha$ for at least one $x$ in each of the $N-1$ intervals $\left\{\left(x_{k}, x_{k+1}\right)\right\}$. We now define $n(y, \alpha)$ as the maximum $N$ for such partitions. ${ }^{3}$ Thus $n(y, \alpha)<\infty$ for every $f(x)$ and $y<\alpha$, and $0 \leqq n(y, \alpha) \leqq n(y)$; indeed, in the absence of local maxima at $f(x)=y$, $0 \leqq n(y, \alpha) \uparrow(1 / 2) n(y)-\epsilon$ as $\alpha \rightarrow y$, where $0 \leqq \epsilon \leqq 1$. It then follows from Theorem 3 that for the general $\beta$-Hölder continuous function $f(x)$, where $0<\beta<1 / 2, n(y, \alpha)$ increases sufficiently rapidly as $\alpha \rightarrow y$ so that

$$
\int_{y}^{B} n(y, \alpha) d \alpha=\infty \text { for every } y, \quad A<y<B ;
$$

where $A=\min f(x)$ and $B=\max f(x)$.

2. A definition and Theorem 1. For a continuous function $f(x)$ $\in C[0,1]$ and a real number $y$, consider the set $\{x: f(x)=y\}$. If it is of cardinality $N$, or is the union of $N$ possibly-degenerate closed intervals, we define $n(y, f)=N$; otherwise, $n(y, f)=\infty$. If the function $f(x)$ is understood, we will shorten $n(y, f)$ to $n(y)$. To avoid certain inconveniences, we will also decrease the value of $n(y)$ by one for $y=f(0)$ or $y=f(1)$; for periodic $f(x)$ this is equivalent to assuming that $f(x)$ is defined on the unit circle. We now prove an important continuity property of $n(y)$, which is slightly weaker than the classical condition of lower semicontinuity. While $n(y)$ in general is not lsc, most of the properties that follow from lsc are implied by (2), although perhaps in a weaker form. For example, the set $\{y: n(y) \leqq N\}$

3 The function $n(y, \alpha)$ was introduced (essentially) in [3]; indeed, if $N\left(E_{y, \alpha}\right)$ is defined as in $\left[3\right.$, p. 591], then $n(y, \alpha+) \leqq N\left(E_{y, \alpha}\right)+1 \leq n(y, \alpha)+1$. 
can differ from a closed set by at most a countable set; see also Corollary 2.3.

Lemma 2.1. Assume $b_{k}-a_{k} \rightarrow 0$ and $a_{c}<y<b_{k}$, where $y$ is some real number and $f(x) \in C[0,1]$. Then,

$$
n(y) \leqq \liminf _{t \rightarrow \infty} \max \left\{n\left(a_{k}\right), n\left(b_{k}\right)\right\} .
$$

Proof. First, assume $0<x_{1}<x_{2}<\cdots<x_{N}<1$ are the $N$ solutions of $f(x)=y$, where $n(y)=N<\infty$. Choose $\epsilon<(1 / 4) \min \left(x_{k+1}-x_{k}\right)$, and $\delta>0$ such that $|f(x)-y| \geqq \delta$ for at least one value of $x$ in each of the $2 N$ intervals $\left(x_{k}-\epsilon, x_{k}\right),\left(x_{k}, x_{k}+\epsilon\right)$. Thus, if $0<\delta_{1}, \delta_{2}<\delta, 2 N \leqq n\left(y-\delta_{1}\right)$ $+n\left(y+\delta_{2}\right)$ by the Intermediate Value Theorem, and for sufficiently large $k$

$$
n(y) \leqq \frac{n\left(a_{k}\right)+n\left(b_{k}\right)}{2} \leqq \max \left\{n\left(a_{k}\right), n\left(b_{k}\right)\right\} .
$$

The case where there are intervals of solutions of $f(x)=y$, or where $y=f(0)$ or $y=f(1)$, can be handled similarly. Likewise, the same argument shows that if $n(y)=\infty$, the right-hand side of (2) cannot be bounded.

REMARK. Similarly, if $y$ is not the ordinate of a local minimum or maximum of $f(x)$ and $y_{k} \rightarrow y$, then $n(y) \leqq \lim \inf _{k \rightarrow \infty} n\left(y_{k}\right)$.

Lemma 2.2. Given a nonconstant $f(x) \in C[0,1]$, assume that $n(y, f)$ $<\infty$ for all $y$ in a set of the second (Baire) category in the range of $f(x)$. Then, there exists a nonempty open interval $(a, b) \subseteq[0,1]$ on which $f(x)$ is of bounded variation.

Proof. Set $E_{N}=\{y: n(y) \leqq N\}$ and $A=\min f(x), B=\max f(x)$. By hypothesis, $\cup E_{N}$ is a set of the second Baire category in the interval $[A, B]$; hence some set $E_{N}$ is dense in some nonempty open interval $(\alpha, \beta) \subseteq[A, B]$. It then follows from Lemma 2.1 that $n(y) \leqq N$ for all $y, \alpha<y<\beta$. Let $(a, b)$ be a component interval of $f^{-1}\{(\alpha, \beta)\}$; I claim that the variation of $f(x)$ on $(a, b)$ is bounded by $N(\beta-\alpha)$. (See also formula (1).) This follows from the fact that if $\sum\left|f\left(x_{k+1}\right)-f\left(x_{k}\right)\right|$ $>N(\beta-\alpha)$ for some partition $a=x_{0}<x_{1}<x_{2}<\cdots<x_{M}=b$, then some open interval $\left(\alpha^{\prime}, \beta^{\prime}\right) \subset[\alpha, \beta]$ must be covered by more than $N$ of the closed intervals $\left\{f\left(\left[x_{k}, x_{k+1}\right]\right)\right\}$.

Corollary 2.3. Assume that $n(y)<\infty$ for every $y$, except perhaps on a set of the first (Baire) category. Then, $n(y)=\infty$ only on a nowhere dense set.

Proof. Assume $A<\alpha<\beta<B$, where $A, B, E_{N}$ are as before. Since 
$U E_{N}$ is of the second category in $[\alpha, \beta]$, some set $E_{N}$ is dense in some interval $\left(\alpha^{\prime}, \beta^{\prime}\right) \subseteq[\alpha, \beta]$, and $n(y) \leqq N$ for $\alpha^{\prime}<y<\beta^{\prime}$ as before. Hence $\{y: n(y)=\infty\}$ is not dense in $(\alpha, \beta)$.

Theorem 1. A ssume $n(y, f)<\infty$ except for a countable number of $y$, or alternately except for a nowhere dense set. Then, there exists a sequence of intervals $\left(a_{n}, b_{n}\right) \subseteq[0,1]$, whose union is dense in $[0,1]$, such that $f(x)$ is of bounded variation in each interval $\left(a_{n}, b_{n}\right)$.

Proof. I claim that every nonempty in terval $(c, d) \subseteq[0,1]$ contains a nonempty interval $(a, b)$ on which $f(x)$ is of bounded variation; this follows by applying Lemma 2.2 to the function $g(x)=f(c+(d-c) x)$. Hence if $\mathcal{F}$ is the collection of all intervals $\left(r_{1}, r_{2}\right)$ with rational endpoints and with $f(x)$ of bounded variation on $\left(r_{1}, r_{2}\right)$, then UF is dense in $[0,1]$.

REMARK. If $f(x)=2 x+x$ sin $1 / x$, then $f(x)$ is of bounded variation on each interval $(1 / n, 1]$ but not on $(0,1]$; thus Theorem 1 cannot be improved even if $n(y, f)<\infty$ for every $y$.

3. Negative results. We recall that a subset $R$ of a complete metric space $E$ is called residual if it is the complement of a set of the first category in $E$; i.e., the complement of a countable union of nowhere dense sets. In particular, a residual set would be nonempty (and even dense in the space), and a countable intersection of residual sets is also residual. In the following, let $C[0,1]$ be the metric space of all continuous functions on $[0,1]$, with the norm $\|f\|=\max _{x}|f(x)|$.

THEOREM 2. For all $f(x)$ in a certain residual set in $C[0,1], n(y, f)$ $=\infty$ for every $y, \min _{x} f(x)<y<\max _{x} f(x)$.

Proof. For $f(x) \in C[0,1]$ and $A=\min f(x), B=\max f(x)$, define

$$
K_{N}=\left\{f: B-A \geqq 2 / N, \inf _{A+1 / N \leqq y \leqq B-1 / N} n(y, f) \leqq N\right\} .
$$

I claim that each set $K_{N}$ is nowhere dense in $C[0,1]$, or, alternately, that any neighborhood of any function $f(x) \in C^{1}[0,1]$ contains an open set which is totally disjoint from $K_{N}$. Choose $f(x) \in C^{1}[0,1]$ and $\eta>0$, and an integer $k$ such that $3 C / k<\eta, 3 C / k<1 / N$, where $C=\max _{x}\left|f^{\prime}(x)\right|$. Thus $|f(l / k)-f((l-1) / k)| \leqq C / k$ for $1 \leqq l \leqq k$; now define

$$
\begin{aligned}
f_{1}(x)= & f_{l-1}+2 C / k \sin 4 \pi N k x, \\
& (l-1) / k \leqq x \leqq(2 l-1) / 2 k, \\
= & f_{l-1}+2 k\left(f_{l}-f_{l-1}\right)(x-(2 l-1) / 2 k), \\
& (2 l-1) / 2 k \leqq x \leqq l / k,
\end{aligned}
$$


where $f_{l}=f(l / k)$. Hence, $\left\|f-f_{1}\right\| \leqq 3 C / k<\eta$, where $\|f\|=\max _{x}|f(x)|$ as above, and $n\left(y, f_{1}\right) \geqq 2 N$ for $\min f_{1}<y<\max f_{1}$. Now, assume $g(x)$ $\in C[0,1],\left\|f_{1}-g\right\|<\epsilon=C / 4 k$. Thus, $f((l-1) / k) \leqq y \leqq f(l / k)$ (or $f_{l} \leqq y$ $\left.\leqq f_{l-1}\right)$, some $l$, for every $y$ in the interval $\min g(x)+2 C / k+\epsilon \leqq y$ $\leqq \max g(x)-2 C / k-\epsilon$; and $n(y, g) \geqq 2 N-1$ for these $y$ by the Intermediate Value Theorem. Hence $K_{N}$ is nowhere dense for any $N$; the residual set required is the complement of $U K_{N}$ plus the constant functions.

The same arguments also apply in the Hölder spaces $C_{0}^{\beta}[0,1]$, $0<\beta<1$. Here $C_{0}^{\beta}[0,1]$ is the closure of $C^{1}[0,1]$ with respect to the norm $\|f\|_{\beta}=\|f\|+\sup _{x, y}|f(x)-f(y)| /|x-y|^{\beta}$; i.e., the set of all $f(x)$ $\in C[0,1]$ such that $f(x)-f(y)=\sigma\left(|x-y|^{\beta}\right)$ uniformly in $x$ and $y .{ }^{4}$

Corollary 3.1. Assume $0<\beta<1$. Then, the set of $f(x) \in C_{0}^{\beta}[0,1]$ such that $n(y, f)=\infty$ for every $y, \min f(x)<y<\max f(x)$, is a residual set in $C_{0}^{\beta}[0,1]$.

Proof. Define $K_{N} \subseteq C_{0}^{\beta}$ as in (3), and note that

$$
\left\|f-f_{1}\right\|_{\beta}=O\left(N^{\beta} / k^{1-\beta}\right) \text {. }
$$

Alternately, since $0 \leqq n(y, \alpha) \leqq n(y)$, where $n(y, \alpha)$ is as defined in the introduction, Corollary 3.1 could be obtained from

Theorem 3. Assume $0<\beta<1$ and $\lambda>\beta /(1-\beta)$. Then, for every $f(x)$ in a certain residual set in $C_{0}^{\beta}[0,1]$, and $A, B$ as before,

$$
\int_{y}^{B} n(y, \alpha)^{\lambda} d \alpha=\infty \text { for all } y, \quad A<y<B .
$$

Proof. For $f(x) \in C_{0}^{\beta}[0,1]$ and $N \geqq 1$, define

$$
K_{N}=\left\{f: B-A \geqq 2 / N, \inf _{A+1 / N \leqq y \leqq B-1 / N} \int_{y}^{B} n(y, \alpha)^{\lambda} d \alpha \leqq N\right\} .
$$

As before, choose $f(x) \in C^{1}[0,1], \eta>0, \delta=1-\beta-\beta / \lambda>0$, $C>\max _{x}\left|f^{\prime}(x)\right|$, and an integer $k$ such that $3 C / k<1 / N, 50 C N / k^{\delta}<\eta$. Define $f_{1}(x)$ as in (4), except with $M=\left[(N k)^{1 / \lambda}\right]$ replacing $N$; thus $\left\|f-f_{1}\right\|_{\beta}=O\left(M^{\beta} / k^{1-\beta}\right)<\eta$. If now $\left\|f_{1}-g\right\|<\epsilon=C / 4 k$, then $g(x)$ satisfies $n(y, y+\epsilon) \geqq M-1$ for $\min g(x)+1 / N \leqq y \leqq \max g(x)-1 / N$, and $\int_{y}^{\beta} n(y, \alpha)^{\lambda} d \alpha \geqq \epsilon(M-1)^{\lambda}>N$ for these $y$ and $C>C(\lambda)$. Hence, $K_{N}$ is nowhere dense in $C_{0}^{\beta}[0,1]$, as before.

Remarks. Assume $A \leqq y<\alpha \leqq B$ and $f(x) \in C_{0}^{\beta}[0,1]$; then $f\left(x_{1}\right)=y$

${ }^{4}$ Indeed, if $f(x)-f(y)=\sigma\left(|x-y|^{\beta}\right)$ and $f(0)=f(1)$, then $\left\|s_{n}-f\right\|_{\beta} \rightarrow 0$, where $\left\{s_{n}(x)\right\}$ are the $(C, 1)$ sums of the trigonometric Fourier series of $f(x)$. 
and $f\left(x_{2}\right)=\alpha$ for some $x_{1}, x_{2}$, and $\alpha-y \leqq\|f\|_{\beta}(1 / n(y, \alpha))^{\beta}$ by the definition of $n(y, \alpha)$. Hence,

$$
n(y, \alpha)=O\left(1 /(\alpha-y)^{1 / \beta}\right)
$$

uniformly in $y$ and $\alpha$, for any $f(x) \in C_{0}^{\beta}[0,1]$. In particular, the integral (5) is always finite for any $\lambda<\beta$. On the other hand, for any $l<1 / \beta-1$, it follows from Theorem 3 that $n(y, \alpha) \neq O\left(1 /(\alpha-y)^{l}\right)$ for any value of $y$ in the interior of the range of a general $f(x) \in C_{0}^{\beta}[0,1]$. As an intermediate result, it can be shown that $n(y, \alpha)$ $\neq O\left(1 /(\alpha-y)^{1 / \beta-\epsilon}\right)$ for any $\epsilon>0$ and all $y$ in a residual set in the range, again for the general $f(x) \in C_{0}^{\beta}[0,1]$.

Actually, there is a close relationship between the behavior of $n(y, \alpha)$ and the modulus of continuity for a function $f(x) \in C[0,1]$, and most of the above results have analogs for more general Banach spaces of continuous functions. For example, let $w(\theta)=\theta \log 1 / \theta$, and let $C_{0}^{w}[0,1]$ be the closure of $C^{1}[0,1]$ with respect to the norm $\|f\|_{w}=\max _{x}|f(x)|+\sup _{x, y}|f(x)-f(y)| / w(|x-y|)$. As before (see footnote 4$), C_{0}^{w}[0,1]$ is identical with the set of $f(x) \in C[0,1]$ such that $f(x)-f(y)=\sigma(w(|x-y|))$ uniformly in $x$ and $y$. An easy adaptation of the proof of Theorem 3 now implies that for all $f(x)$ in a residual set in $C_{0}^{w}[0,1]$,

$$
\int_{y}^{B} \exp n(y, \alpha)^{2} d \alpha=\infty \text { for all } y, \quad A<y<B,
$$

where $A=\min f(x), B=\max f(x)$ as before. In particular, this gives a class of functions $f(x) \in C[0,1]$ with $\|f\|_{\beta}=O(1 /(1-\beta)), 0<\beta<1$, such that $n(y)=\infty$ identically for $A<y<B$.

Indeed, the existence of $f(x) \in C[0,1]$ with $n(y)=\infty$ for $A \leqq y \leqq B$ is part of the folk lore of the subject; see e.g. the result of Kunen in $[3$, p. 601].

\section{REFERENCES}

1. S. Banach, Sur une classe des fonctions continues, Fund. Math. 8 (1926), 166173; Sur les lignes rectifiables et les surfaces dont l'aires finie, ibid. 7 (1925), 225-237.

2. N. Bary, Mémoire sur la représentation finie des fonctions continues, Math. Ann. 103 (1930), 185-248, and 598-653.

3. A. Garsia and S. Sawyer, On some classes of continuous functions with convergent Fourier series, J. Math. Mech. 13 (1964), 589-602.

4. S. Saks, Theory of the integral, Dover, New York, 1964.

California Institute of Technology and NEW YoRK UNIVERSITY 